\title{
Covid 19 Review
}

\section{By \\ Prof. Dr. Ashraf Mohamed Abbas}

Scientific molecular biology consultant members of Magazine Head of Genetic Engineering Research

Department, VSVRI, Cairo, Egypt.

\section{Doi: 10.21608/asajs.2020.88549}

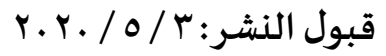

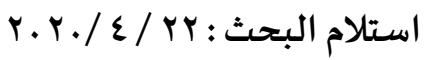

\section{Introduction}

Coronaviruses (CoVs) are a viruses group that co-infects humans and other vertebrate animals. CoV infections affect the respiratory, gastrointestinal, liver, and central nervous systems of humans, livestock, birds, bats, mice, and many other wild animals [Wang, et.al, 2006, Ge].

The source of unexplained pneumonia was first discovered in Wuhan in Dec, 2019, and SARS-CoV-2, a new coronavirus, was isolated from the respiratory epithelium of patients. It belongs to a new evolutionary branch within the $\mathrm{CoV}$. On Feb. 11th, 2020, the new coronavirus was officially renamed "SARS-CoV-2" from "2019-nCoV" [Gorbalenya et.al,2020]. The disease caused by SARS-CoV-2 was called "coronavirus disease 2019" (COVID-19) [Who 2020]. Coronavirus Genetics

Coronaviruses, they look like halos (known as coronas) when viewed under the electron microscope, are a large family of RNA viruses. The typical generic coronavirus genome is a single strand of RNA, 32 kilo bases long, and is the largest known RNA virus genome. Coronaviruses have the highest 


\section{Doi:10.21608/asajs.2020.88549 Dr. Ashraf Mohamed Abbas}

known recombination frequency of any positive-strand RNA virus, promiscuously combining genetic information from different sources when a host is infected with multiple coronaviruses. In other words, these viruses mutate and change at a high rate, which can create havoc for both diagnostic detection as well as therapy (and vaccine) regimens.

Coronaviruses have an unusual replication process, which involves a 2-step replication mechanism. Many RNA virus genomes contain a single open reading frame (ORF) which is then translated as a single polyprotein that is then catalytically cleaved into smaller functional viral proteins, but coronaviruses can contain up to 10 separate ORFs. Most ribosomes translate the biggest one of these ORFs, called replicase, which alone is twice the size of many other RNA viral genomes. The replicase gene encodes a series of enzymes that use the rest of the genome as a template to produce a set of smaller, overlapping messenger RNA molecules, which are then translated into the structural proteins -- the building blocks of new viral particles. [asm.org 2020]

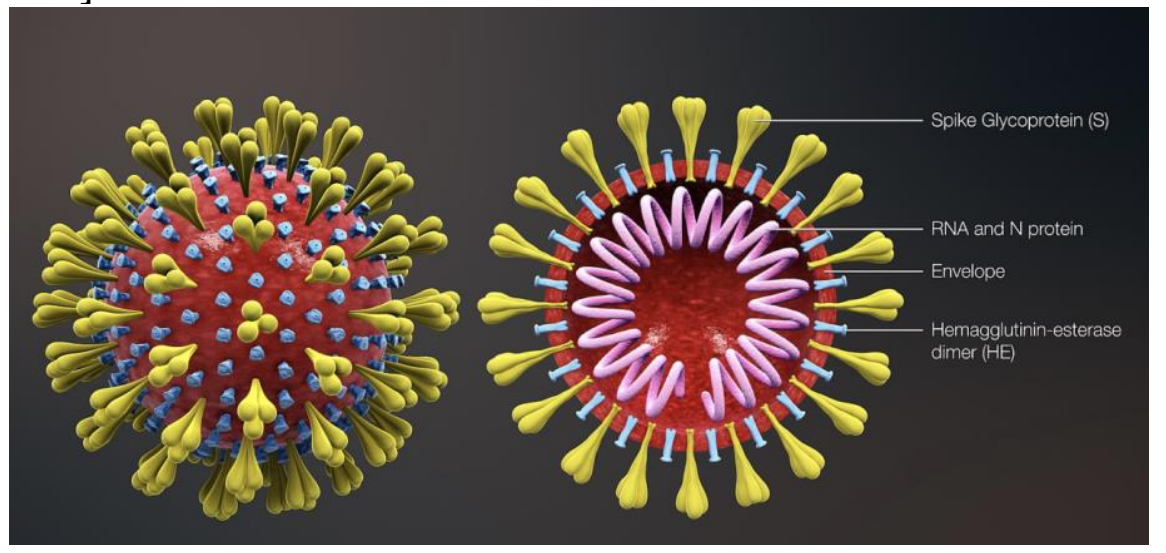

Figure (1) morphology of the Covid 19 History and origin [Kumar etal,2020]

Corona virus was reported as cold in 1960. Canadian study showed that, nearly five hundred patients were diagnosed $r$ 
as Flu-like system in 2001. Seventeen to eighteen cases of them were confirmed as infected with corona virus strain by polymerase chain reaction(PCR). Corona was treated as simple non-fatal virus till 2002.

In 2003, various reports published with the proofs of spreading the corona to many countries such as United States America, Hong Kong, Singapore, Thailand, Vietnam and in Taiwan. Several case of severe acute respiratory syndrome caused by corona and their mortally more than 1000 patient was reported in 2003. This was terrible event for microbiologist. When microbiologist was started insisted on these problems. After a deep efforts they conclude and understand the pathogenesis of disease and discovered as corona virus.

But till total 8096 patient was confirmed as infected with corona virus. So in 2004, World health organization and centers for disease control and prevention declared as "state emergency". Hong Kong study report was confirmed five patient of severe acute respiratory syndrome while thirty of them were confirmed as corona virus infected. In 2012, Saudi Arabian reports were presented several infected patient and deaths [CDC 2003 and WHO 2003] COVID-19 was first identified and isolated from pneumonia patent belongs to Wuhan, china [Who 2020] 


\section{Doi:10.21608/asajs.2020.88549 Dr. Ashraf Mohamed Abbas}

\section{Covid 19 symptoms figure (2)}

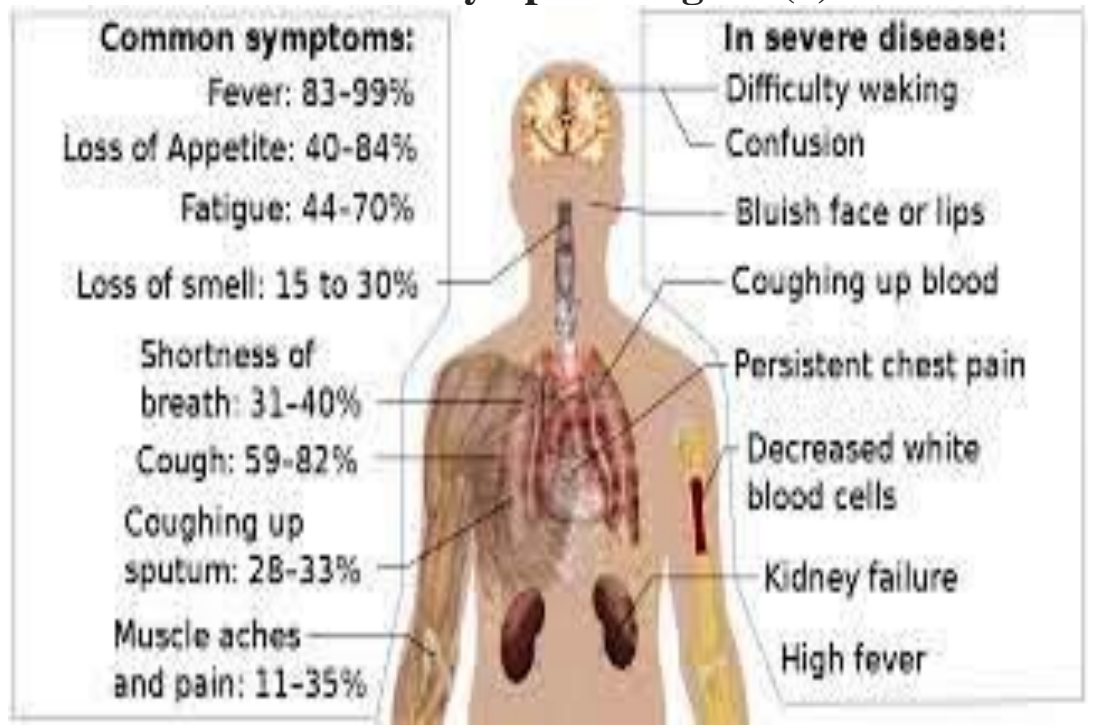

Covid 19 symptoms) [Mayo Clinic 2020]

Common symptoms include fever, cough, fatigue, shortness of breath and loss of smell. While the majority of cases result in mild symptoms, some progress to viral pneumonia, multi-organ failure, or cytokine storm. More concerning symptoms include difficulty breathing, persistent chest pain, confusion, difficulty waking, and bluish skin. The time from exposure to onset of symptoms is typically around five days but may range from two to fourteen day.

\section{Spreading nature}

the infection can get through close contact with a person who has symptoms from the virus includes cough and sneezing. Generally corona virus was spread via air-borne zoonotic droplets. Virus was replicated in ciliated epithelium that caused cellular damage and infection at infection site. 


\section{المجلة العربية للعلوم الزراعية}

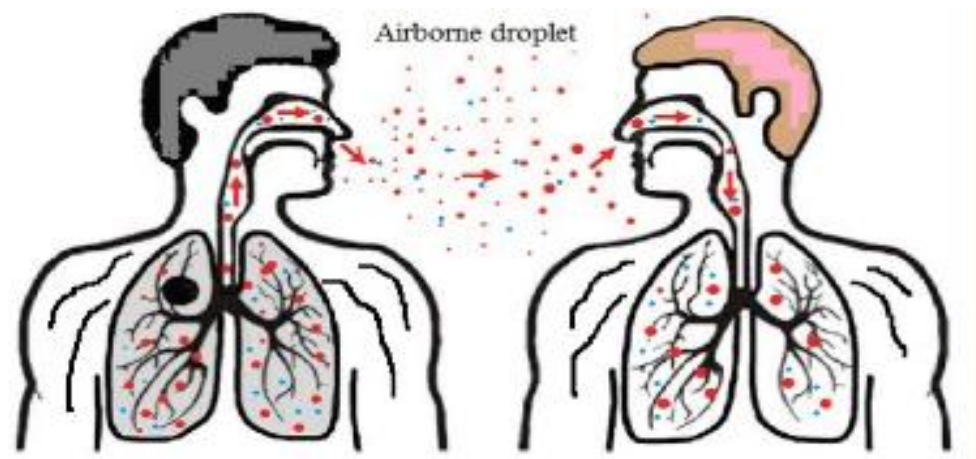

Figure (3) Corona virus transmission via airborne droplets
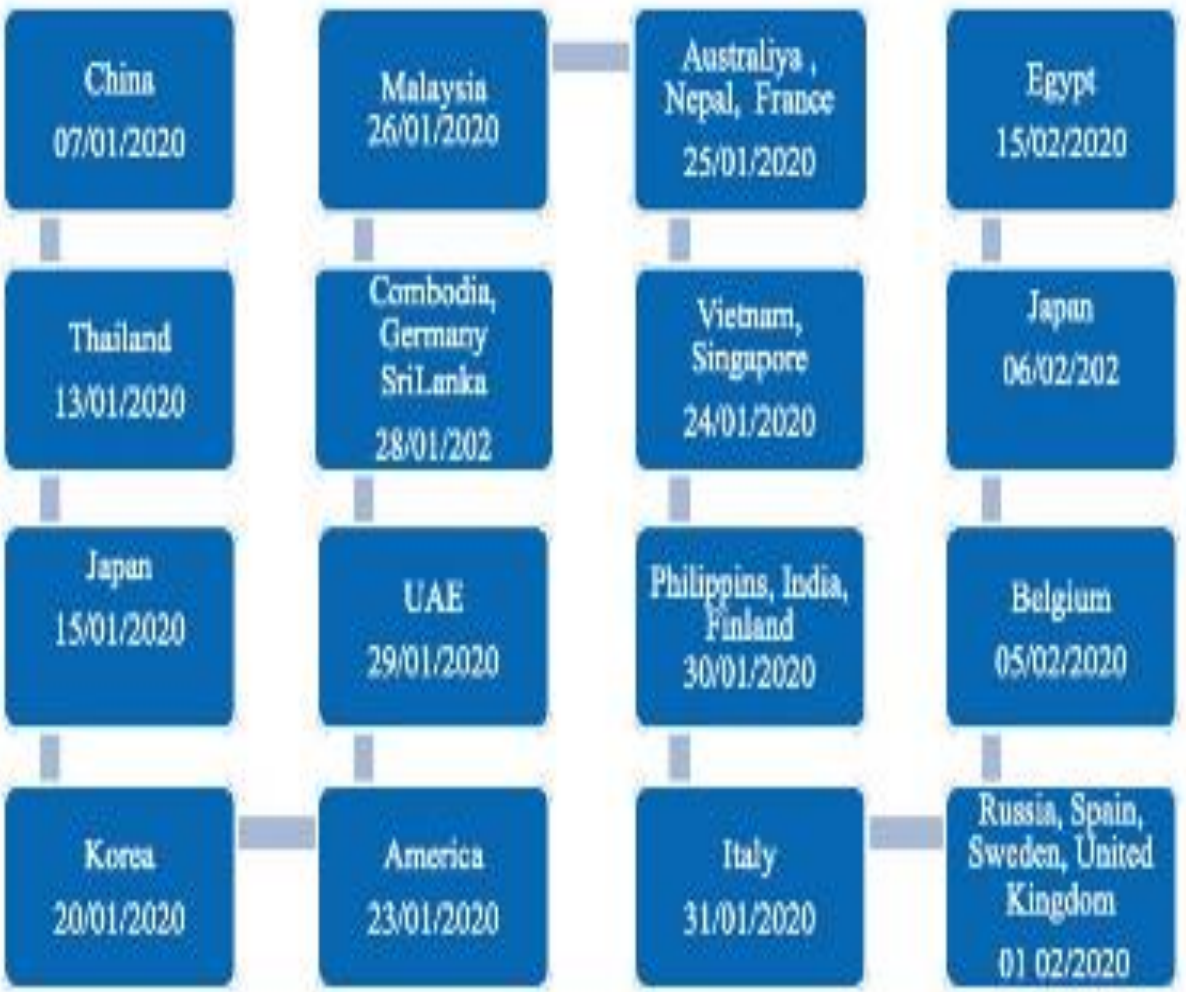

Figure (4) History of spreading of 2019 n-cov.

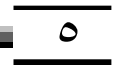




\section{Doi:10.21608/asajs.2020.88549 Dr. Ashraf Mohamed Abbas}

\section{The spreading History of 2019 n-cov. [Kumar etal,2020]}

31 Dec. 2019, China, East Asia, most populated coun-try in world was informed to WHO regarding pneumonia cases with unknown etiology. Until 3 Jan. 2020 a total of 44 pneumonia cases were detected. On 7 Jan2020, Chinese research authorities were announced that they were isolated new virus from sea food market in Wuhan city; Named as 2019-nCoV. On 13 Jan. 2020 Ministry of public health Thailand were reported one patient imported from Wuhan, China. On 15 Jan. 2020, the ministry of health, labor and welfare Japan were reported first case imported from Wuhan China. On 20 Jan. 2020, National IHR Focal point from the Korea was reported first case 2019-nCoV in Korea. On 23 Jan. 2020, United State of America were confirmed first case of 2019-nCoV in America. On 24 Jan. 2020, Vietnam has reported First case of 2019-nCoV with not travel history from China, while his family member was the China traveler. So it's the first incidence of human to human transmission of corona virus. On 24 Jan. 2020, the government of Singapore was confirmed First case of 2019-nCoV. On 25 Jan. 2020, the government of Australia, federal democratic republic of Nepal and French republic were confirmed first of 2019-nCoV. Other countries also were detected and re-ported the cases of 2019nCoV as On, 26 Jan. 2020 (Malaysia), 27 Jan. 2020 (Canada), 28 Jan. 2020 (Cambodia, Germany, Sri Lanka), 29 Jan. 2020 (United Arab Emirates), 30 Jan. 2020 (Philippines, India , Finland), 31 Jan. 2020 (Italy), 1 Feb. 2020 (Russian Federation, Spain, Sweden, United Kingdom), 5 Feb. 2020 (Belgium), 6 Feb. 2020 (Japan), 15 Feb. 2020 (Egypt)

\section{Phylogenetic SARS-CoV-2 genomes. .[Forster, et.al, 2020]}

In a phylogenetic network analysis of 160 complete human severe acute respiratory syndrome coronavirus 2 (SARSCov-2) genomes, three central variants distinguished by amino

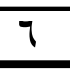


acid changes, which we have named $\mathrm{A}, \mathrm{B}$, and $\mathrm{C}$, with $\mathrm{A}$ being the ancestral type according to the bat outgroup coronavirus. The $\mathrm{A}$ and $\mathrm{C}$ types are found in significant proportions outside East Asia, that is, in Europeans and Americans. In contrast, the B type is the most common type in East Asia, and its ancestral genome appears not to have spread outside East Asia without first mutating into derived B types, pointing to founder effects or immunological or environmental resistance against this type outside Asia. The network faithfully traces routes of infections for documented coronavirus disease 2019 (COVID-19) cases, indicating that phylogenetic networks can likewise be successfully used to help trace undocumented COVID-19 infection sources, which can then be quarantined to prevent recurrent spread of the disease worldwide.(Forster, et.al, 2020).

\section{Significance}

This is a phylogenetic network of SARS-CoV-2 genomes sampled from across the world. These genomes are closely related and under evolutionary selection in their human hosts, sometimes with parallel evolution events, that is, the same virus mutation emerges in two different human hosts. This makes character-based phylogenetic networks the method of choice for reconstructing their evolutionary paths and their ancestral genome in the human host. The network method has been used in around 10,000 phylogenetic studies of diverse organisms, and is mostly known for reconstructing the prehistoric population movements of humans and for ecological studies, but is less commonly employed in the field of virology. 


\section{Doi:10.21608/asajs.2020.88549 Dr. Ashraf Mohamed Abbas}

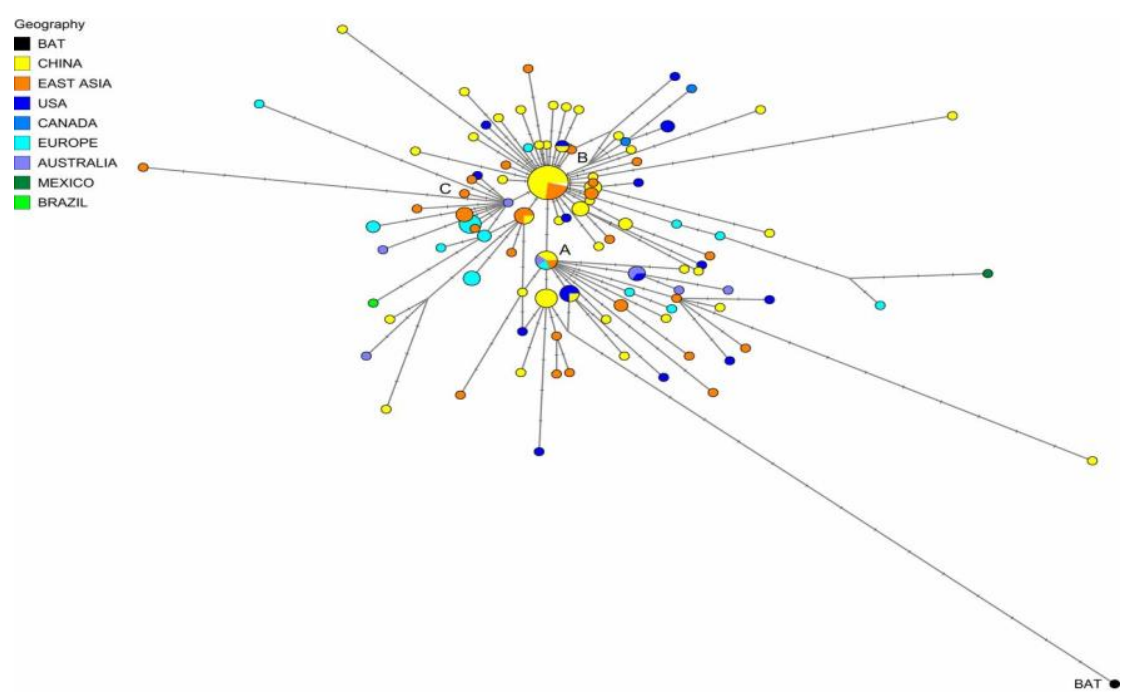

Peter Forster et al. PNAS doi:10.1073/pnas.2004999117

\section{Figure(5) Phylogenetic network of 160 SARS-CoV-2} genomes. Node $\mathrm{A}$ is the root cluster obtained with the bat $(R$. affinis) coronavirus isolate BatCoVRaTG13 from Yunnan Province. Circle areas are proportional to the number of taxa, and each notch on the links represents a mutated nucleotide position. The sequence range under consideration is 56 to 29,797 , with nucleotide position (np) numbering according to the Wuhan 1 reference sequence. The median-joining network algorithm and the Steiner algorithm were used, both implemented in the software package Network5011CS (https://www.fluxus-engineering.com/), with the parameter epsilon set to zero, generating this network containing 288 mostparsimonious trees of length 229 mutations. The reticulations are mainly caused by recurrent mutations at np11083. The 161 taxa (160 human viruses and one bat virus) yield 101 distinct genomic sequences.

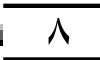




\section{Diagnosis of Covid 19(Wikipedia 2020)}

The WHO has published several testing protocols for the disease. The standard method of testing is real-time reverse transcription polymerase chain reaction (rRT-PCR). The test is typically done on respiratory samples obtained by a nasopharyngeal swab; however, a nasal swab or sputum sample may also be used. Results are generally available within a few hours to two days. Blood tests can be used, but these require two blood samples taken two weeks apart, and the results have little immediate value. Chinese scientists were able to isolate a strain of the coronavirus and publish the genetic sequence so laboratories across the world could independently develop polymerase chain reaction (PCR) tests to detect infection by the virus. As of 4 April 2020, antibody tests (which may detect active infections and whether a person had been infected in the past) were in development, but not yet widely used. The Chinese experience with testing has shown the accuracy is only 60 to $70 \%$. The FDA in the United States approved the first point-of-care test on 21 March 2020 for use at the end of that month.

Diagnostic guidelines released by Zhongnan Hospital of Wuhan University suggested methods for detecting infections based upon clinical features and epidemiological risk. These involved identifying people who had at least two of the following symptoms in addition to a history of travel to Wuhan or contact with other infected people: fever, imaging features of pneumonia, normal or reduced white blood cell count, or reducedlymphocyte count. A study asked hospitalised COVID-19 patients to cough into a sterile container, thus producing a saliva sample, and detected the virus in eleven of twelve patients using RT-PCR. This technique has the potential 


\section{Doi:10.21608/asajs.2020.88549 Dr. Ashraf Mohamed Abbas}

of being quicker than a swab and involving less risk to health care workers (collection at home or in the car).

Along with laboratory testing, chest CT scans may be helpful to diagnose COVID-19 in individuals with a high clinical suspicion of infection but are not recommended for routine screening. Bilateral multilobar ground-glass opacities with a peripheral, asymmetric, and posterior distribution are common in early infection Subpleural dominance, crazy paving (lobular septal thickening with variable alveolar filling), and consolidation may appear as the disease progresses.

In late 2019, WHO assigned the emergency ICD-10 disease codes U07.1 for deaths from lab-confirmed SARS-CoV-2 infection and U07.2 for deaths from clinically or epidemiologically diagnosed COVID-19 without lab-confirmed SARS-CoV-2 infection.

\section{Interpretation of Molecular Results.}

In the United States, initially if all two targets (nucleocapsid proteins $\mathrm{N} 1$ and $\mathrm{N} 2$ ) test positive, a case is considered to be laboratory confirmed . A cycle threshold value (Ct-value) less than 40 is defined as a positive test, while 252 a $\mathrm{Ct}$-value of 40 or more is defined as a negative test. A Ct -value (Holshue etal 2020)

\section{COVID-19 vaccine trial}




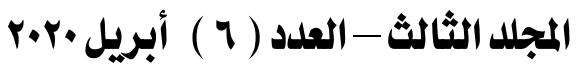

\section{المجلة العربية للعلور الزراعية}

\section{COVID-19 Oxford Vaccine Trial}

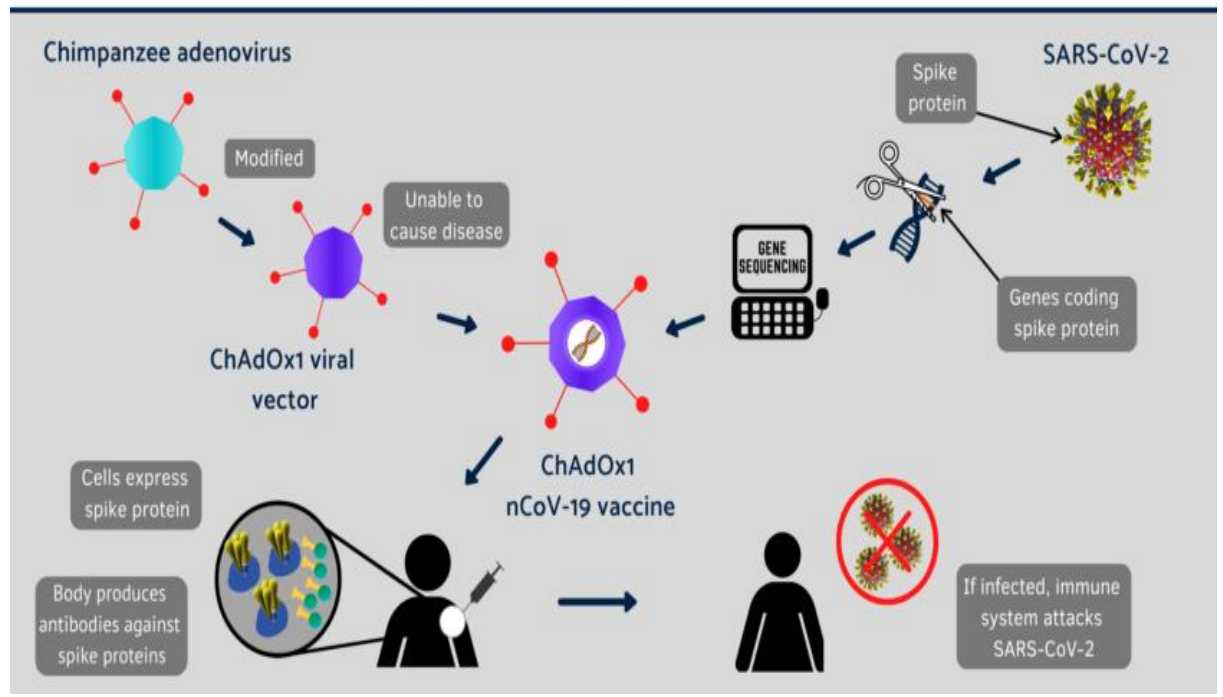

University of Oxford researchers have begun testing a COVID19 vaccine in human volunteers in Oxford 23 April 2020. Around 1,110 people will take part in the trial, half receiving the vaccine and the other half (the control group) receiving a widely available meningitis vaccine. Of the first two volunteers to take part April 29 2020, one will likewise receive the vaccine and the other the control. The researchers started screening healthy volunteers (aged 18-55) in March for their upcoming ChAdOx1 nCoV-19 vaccine trial in the Thames Valley Region. The vaccine is based on an adenovirus vaccine vector and the SARSCoV-2 spike protein, and has been produced in Oxford.

\section{Oxford Vaccine Centre COVID-19 Phase I Clinical Trial Explained}

The study is to test a new vaccine against COVID-19 in healthy volunteers. It aims to assess whether healthy people can be protected from COVID-19 with this new vaccine called ChAdOx1 nCoV-19. It will also provide valuable information on 


\section{Doi:10.21608/asajs.2020.88549 Dr. Ashraf Mohamed Abbas}

safety aspects of the vaccine and its ability to generate good immune responses against the virus.

ChAdOx1 nCoV-19 is made from a virus (ChAdOx1), which is a weakened version of a common cold virus (adenovirus) that causes infections in chimpanzees, that has been genetically changed so that it is impossible for it to grow in humans. Genetic material has been added to the ChAdOx1 construct, that is used to make proteins from the COVID-19 virus (SARS-CoV-2) called Spike glycoprotein (S). This protein is usually found on the surface of SARS-CoV-2 and plays an essential role in the infection pathway of the SARS-CoV-2 virus. The SARS-CoV-2 coronavirus uses its spike protein to bind to ACE2 receptors on human cells to gain entry to the cells and cause an infection.

By vaccinating with ChAdOx $1 \mathrm{nCoV}-19$, we are hoping to make the body recognize and develop an immune response to the Spike protein that will help stop the SARS-CoV-2 virus from entering human cells and therefore prevent infection. Vaccines made from the ChAdOx1 virus have been given to more than 320 people to date and have been shown to be safe and well tolerated, although they can cause temporary side effects, such as a temperature, headache or sore arm. Up to 1102 participants will be recruited across multiple study sites in Oxford, Southampton, London and Bristol. These participants will be randomly allocated to receive either the ChAdOx1 nCoV-19 vaccine or a licensed vaccine (MenACWY) that will be used as a 'control' for comparison. At the start of the trial we will also recruit a separate small group of 10 volunteers who will receive 2 doses of ChAdOx1 nCoV-19 four weeks apart. The MenACWY vaccine is a licensed vaccine against group $\mathrm{A}, \mathrm{C}, \mathrm{W}$ and $\mathrm{Y}$ meningococcus which has been given routinely to teenagers in the UK since 2015 and protects against one of the most common

$T$ 
causes of meningitis and sepsis. This vaccine is also given as a travel vaccine for high risk countries.

The MenACWY vaccine is being used as an 'active control' vaccine in this study, to help us understand participants' response to ChAdOx1 nCoV-19. The reason for using this vaccine, rather than a saline control, is because we expect to see some minor side effects from the ChAdOx1 nCOV-19 vaccine such as a sore arm, headache and fever. Saline does not cause any of these side effects. If participants were to receive only this vaccine or a saline control, and went on to develop side effects, they would be aware that they had received the new vaccine. It is critical for this study that participants remain blinded to whether or not they have received the vaccine, as, if they knew, this could affect their health behavior in the community following vaccination, and may lead to a bias in the results of the study. Participants must: Be aged 18-55 years old, be in good health, and be based in one of the recruiting areas.

Participants must NOT: Have tested positive for COVID-19, be pregnant, intending to become pregnant, or breastfeeding during the study, or have previously taken part in a trial with an adenoviral vaccine or received any other coronavirus vaccines.

The main focus of the study is to find out if this vaccine is going to work against COVID-19, if it won't cause unacceptable side effects and if it induces good immune responses. The dose used in this trial was chosen based on previous experiences with other ChAdOx1 based vaccines.

Study participants will not know whether they have received the ChAdOx1 $\mathrm{nCoV}-19$ vaccine until the end of the trial. Participants will be given an E-diary to record any symptoms experienced for 7 days after receiving the vaccine. They will also record if they feel unwell for the following three weeks. 


\section{Doi:10.21608/asajs.2020.88549 Dr. Ashraf Mohamed Abbas}

Following vaccination, participants will attend a series of followup visits. During these visits, the team will check participants' observations, take a blood sample and review the competed Ediary. These blood samples will be used to assess the immune response to the vaccine.

If participants develop COVID-19 symptoms during the study, they can contact a member of the clinical team, and we will assess them to check whether they have become infected with the virus. If a participant was very unwell, we would call our colleagues in the hospital and ask them to review the volunteer if appropriate.

To assess whether the vaccine works to protect from COVID-19, the statisticians in our team will compare the number of infections in the control group with the number of infections in the vaccinated group. For this purpose, it is necessary for a small number of study participants to develop COVID-19. How quickly we reach the numbers required will depend on the levels of virus transmission in the community. If transmission remains high, we may get enough data in a couple of months to see if the vaccine works, but if transmission levels drop, this could take up to 6 months.

A high proportion of vaccines are found not to be promising even before clinical trials. Moreover, a significant proportion of vaccines that are tested in clinical trials don't work. If we are unable to show that the vaccine is protective against the virus, we would review progress, examine alternative approaches, such as using different numbers of doses, and would potentially stop the programme.

We plan to vaccinate 800 volunteers in the UK over the next month. If the trial is successful in the UK, then the Oxford team will approach scientists in the Kenya Medical Research Institute (KEMRI) and will approach the Government of Kenya for permission to evaluate in Kenya. 
In Germany, BioNTech and Pfizer are planning on carrying out clinical evaluation of the first four vaccine candidates to come out of their global "Lightspeed" COVID-19 vaccine development program. Each candidate represents a different mRNA format and target antigen. Two of the four vaccine candidates are nucleoside modified mRNA (modRNA) vaccines, a third is a uridine containing mRNA (uRNA), and the fourth vaccine candidate utilizes self-amplifying mRNA (saRNA). Each mRNA format is combined with a lipid nanoparticle (LNP) formulation. Two of the candidates incorporate the larger spike sequence, and the other two encode the smaller optimized receptor-binding domain (RBD) from the spike protein. The RBD-based candidates contain the piece of the spike that is thought to be most important for eliciting antibodies that can inactivate the virus. The first Phase I/II dose-ranging study in Germany will evaluate the effects of repeat immunization for three of the four vaccine candidates that utilize uRNA or modRNA. "We are pleased to have completed preclinical studies in Germany and to have received this important regulatory approval to initiate this first-in-human trial," stated Ugur Sahin, CEO and co-founder of BioNTech. "The speed with which we were able to move from the start of the program to regulatory approval to initiate this first-in-human trial speaks to the high level of engagement from everyone involved." 


\section{Doi:10.21608/asajs.2020.88549 Dr. Ashraf Mohamed Abbas}

\section{References}

Www.https://asm.org/Articles/2020/January/2019-Novel

Coronavirus-2019-nCoV-Update-Uncoating

Centers for Disease Control and Prevention (CDC). Update: Outbreak of severe acute respiratory syndrome-worldwide, 2003. MMWR Morb Mortal Wkly Rep. 2003;52(12):241-6.2.

Chen, Y.; Guo, D. Molecular mechanisms of coronavirus RNA capping and methylation. Virol. Sin. 2016, 31, 3-11.

Ge, X.-Y.; Li, J.; Yang, X.-L.; Chmura, A.; Zhu, G.; Epstein, J.H.; Mazet, J.K.; Hu, B.; Zhang, W.; Peng, C.; et al 2013. Isolation and characterization of a bat SARS-like coronavirus that uses the ACE2 receptor. Nature 2013, 503, 535-538.

Gorbalenya, A.E.; Baker, S.C.; Baric, R.S.; de Groot, R.J.; Drosten, C.; Gulyaeva, A.A.; Haagmans, B.L.; Lauber, C.; Leontovich, A.M.; Neuman, B.W.; et al. 2020 Severe acute respiratory syndrome-related coronavirus: The species and its viruses-A statement of the Coronavirus Study Group. bioRxiv 2020.

Holshue ML, DeBolt C, Lindquist S, Lofy KH, Wiesman J, Bruce H, Spitters C, Ericson K, Wilkerson S, Tural A, Diaz G, Cohn A, Fox L, Patel A, Gerber SI, Kim L, Tong S, Lu X, Lindstrom S, Pallansch MA, Weldon WC, Biggs HM, Uyeki TM, Pillai SK. 2020. First Case of 2019 Novel Coronavirus in the United States. N Engl J Med 382:929 -93

Kumar D, Malviya R, Kumar Sharma P. Corona Virus: A Review of COVID-19. EJMO 2020;4(1):8-25.

Wang, L.F.; Shi, Z.; Zhang, S.; Field, H.; Daszak, P.; Eaton, B.T. Review of bats and SARS. Emerg. Infect. Dis.2006, 12, 1834. 
World Health Organization (WHO 2003). Coronavirus never before seen in humans is the cause of SARS- update 31 . Geneva: The Organization; 2003

World Health Organization (WHO jan.2020) WHO Statement Regarding Cluster of Pneumonia Cases in Wuhan, China Geneva 2020 [up-dated 9 January 2020 and 14 January 2020]. Available from:

https://www.who.int/china/news/detail/09-01-2020-whostatement-regarding-cluster-of-pneumoniacases-in-wuhanchina

World Health Organization (WHO Feb.2020) Named the New Pneumonia

“COVID19".Availableonline: http://www.xinhuanet.com/ world/202002/12/c 1125561389.htm (accessed on 12 February 2020).

Mayo Clinic. 2020 "Coronavirus disease 2019 (COVID-19)Symptoms and causes", USA. Retrieved 14April 2020https://en.wikipedia.org/wiki/C oronavirus_disease_2019

Zhu N, Zhang D, Wang W, Li X, Yang B, Song J, et al. (2020) A Novel Coronavirus from Patients with Pneumonia in China, 2019. 24 January 2020. New England Journal of Medicine. 
Doi:10.21608/asajs.2020.88549 Dr. Ashraf Mohamed Abbas 